\title{
Complicaciones maternas de la cesárea en gestantes a término en periodo expulsivo en un hospital general de Lima, Perú
}

\author{
Maternal complications of cesarean sections performed in the expulsive period of labor in a general hospital \\ in Lima, Peru
}

Javier Puma ${ }^{1, a, 2, e}$, Jorge Díaz ${ }^{1, a, 2, b, d,}$, Carlos Caparó 1,a,2,c

\section{RESUMEN}

Objetivo: Describir las complicaciones maternas de la cesárea en gestantes a término en periodo expulsivo en un hospital general. Material y métodos: Estudio descriptivo, observacional, retrospectivo, tipo serie de casos, realizado en el Hospital Nacional Cayetano Heredia entre el 1 de enero de 2011 hasta el 31 diciembre de 2012. Se incluyeron 67 gestantes a término operadas en periodo expulsivo. Se revisaron las historias clínicas de las pacientes, se registraron las características clínicas y las complicaciones maternas. Resultados: En el periodo de estudio se realizaron 4218 cesáreas, siendo el 1,84\% hechas en periodo expulsivo. En general, 59/ 67 (88,1\%) presentaron algún tipo de complicación. Se observaron 8 casos de hipotonía uterina $(11,9 \%)$ y $2(2,9 \%)$ de atonía. En $5(7,4 \%)$ pacientes ocurrieron desgarros de segmento adyacente a la histerotomía, siendo 2 de ellos asociados a compromiso de cuerpo uterino y vagina, y $4(5,9 \%)$ casos a laceración de arteria uterina, uno fue bilateral. No se produjeron lesiones en tracto urinario ni digestivo y tampoco hubo necesidad de reintervención quirúrgica. Cuatro pacientes presentaron hemorragia puerperal, dos asociados a hipotonía uterina post cesárea. En $56(83,5 \%)$ casos ocurrió anemia post operatoria. Hubo $1(1,5 \%)$ caso de endometritis, $3(4,5 \%)$ infecciones de herida operatoria. Conclusiones: La cesárea realizada en período expulsivo ocasiona complicaciones frecuentes, algunas muy severas.

PALABRAS CLAVE: Complicaciones del embarazo, cesárea, segundo periodo del trabajo de parto. (Fuente: DeCS BIREME).

\section{SUMMARY}

Objective: To determine the maternal complications of cesarean sections performed in the expulsive period of labor in a general hospital. Methods: Case series performed at Hospital Nacional Cayetano Heredia from January 1st, 2011 to December 31st, 2012. Sixty-seven pregnants in whom a cesarean section was performed during the expulsive period of labor were included. Clinical charts were reviewed to gather clinical information. Results: A total of 4218 cesarean sections were performed during the study period, $1.84 \%$ were performed during the expulsive

\footnotetext{
1 Departamento de Ginecología y Obstetricia, Hospital Nacional Cayetano Heredia. Lima, Perú.

2 Facultad de Medicina Alberto Hurtado, Universidad Peruana Cayetano Heredia. Lima, Perú.

a Médico Gineco-Obstetra;

b Doctor en Medicina;

c Profesor Auxiliar;

d Profesor Principal

e Ex Médico residente
} 
period of labor. Overall, 88.1\% (59/67) had any kind of complication. Eight cases of uterine hypotonia (11.9\%) were observed, and 2 of uterine atonia (2.9\%). Detachment of the segment close to the uterine incision was observed in 5 patients (7.4\%), in two cases affection of the vagina and uterus was observed, and 4 cases $(5.9 \%)$ of laceration of the uterine artery, one with bilateral laceration, were observed. No urinary tract or digestive tract lesions were observed; there was also no need to perform re-interventions. Four patients presented puerperal hemorrhage; two were associated with uterine hypotonia. Post-operative anemia was observed in 56 patients $(83.5 \%)$. There was one case $(1.5 \%)$ of endometritis and $3(4.5 \%)$ of wound infections. Conclusions: Cesarean sections performed in the expulsive period of labor are associated with frequent complications; some of them are very severe.

KEY WORDS: Pregnancy complications, cesarean section, second labor stage. (Source: MesH NLM).

\section{INTRODUCCIÓN}

La Cesárea es el procedimiento quirúrgico más común realizado en los Estados Unidos, y la primera indicación es la desproporción feto-pélvica. Las técnicas utilizadas durante este procedimiento a menudo varían de manera significativa entre los ginecólogos $(1,2)$.

El parto obstruido es definido por la Organización Mundial de la Salud (OMS) como trabajo de parto en que la parte de presentación del feto no puede progresar en el canal del parto, a pesar de contracciones uterinas adecuadas (3).

El porcentaje de cesáreas en segunda etapa de trabajo de parto está en aumento. La extracción de la cabeza fetal encajada por cesárea es una experiencia temida por las complicaciones que pueden producirse y la falta de difusión de las técnicas adecuadas para prevenirlas (4-9). Las complicaciones más frecuentes de la cesárea en general son la infección de herida, la necesidad de transfusión de sangre, la hemorragia, la endomiometritis y las lesiones viscerales y fetales (10).

Las mujeres que son sometidas a cesárea durante la segunda etapa del trabajo tienen un aumento de la morbilidad materna y fetal y requieren de una atención especial (11-15). En la literatura, se discuten estrategias para mejorar los resultados maternos perinatales en pacientes en trabajo de parto, una de ellas es lograr un tiempo que no exceda 30 minutos entre la decisión de cirugía y la intervención quirúrgica (16).

La cesárea es importante para disminuir la morbimortalidad materno fetal, sin embargo las complicaciones que pueden ser mayores y más frecuentes en período expulsivo debemos conocerlas para planificar mejor nuestras cirugías.
El estudio tuvo como objetivo describir las complicaciones maternas de la cesárea en gestantes a término en periodo expulsivo.

\section{MATERIAL Y MÉTODOS}

Estudio descriptivo, observacional, retrospectivo, tipo serie de casos, realizado en el Hospital Nacional Cayetano Heredia del 1 de enero del 2011 al 31 diciembre del 2012.

Criterios de inclusión.

- Pacientes gestantes a término a quienes se les realizó cesárea durante el periodo expulsivo

- Trabajo de parto controlado

Tabla 1. Características demográficas y obstétricas.

\begin{tabular}{lcr}
\hline & n & \% \\
\hline EDAD & 18 & 26,9 \\
$\quad$ Menor de 19 años & 39 & 58,2 \\
19 - 35 años & 10 & 14,9 \\
35 años o mayor & & \\
ESTADO CIVIL & 10 & 14,9 \\
$\quad$ soltera & 6 & 9,0 \\
$\quad$ casada & 51 & 76,1 \\
conviviente & & \\
GESTACIÓN PREVIA & 35 & 52,2 \\
$\quad$ Ninguna & 26 & 38,8 \\
1 ó 2 & 6 & 9,0 \\
3 o más & & \\
PARIDAD & & 64,2 \\
nulípara & 43 & 35,8 \\
1 a más partos previos & 24 &
\end{tabular}


- Feto en presentación cefálica

- Pacientes sin Ruptura prematura de membranas.

Criterios de exclusión

- Cesárea anterior

- Intento de parto instrumentado

- Diagnóstico o sospecha de corioamnionitis

- Sufrimiento fetal

- Historia clínica extraviada.

- Antecedente de cirugía abdominal previa.

Ruptura prematura de membranas fue definida como la ruptura de las membranas que se produce antes del inicio de labor departo (17).

Tabla 2. Características peri-operatorias.

\begin{tabular}{lll}
\hline n & $\%$
\end{tabular}

\section{Tiempo de ruptura de membrana}

$$
\begin{aligned}
& \leq 16 \text { horas } \\
& >16 \text { horas }
\end{aligned}
$$

Número de tactos vaginales

$\begin{array}{ccc}\leq 4 & 9 & 13,4 \\ >5 & 58 & 86,6\end{array}$

Tiempo periodo expulsivo (min)

$$
9
$$

120-180

22

$180-240$

$240-320$

30

6

Altura de presentación final

$\begin{array}{lr}\text { Flotante } & 1 \\ -4 & 4 \\ -3 & 1 \\ -2 & 1 \\ -1 & 1 \\ 0 & 1 \\ +1 & 6 \\ +2 & 3\end{array}$

Antibiótico profiláctico

Cefazolina

Clindamicina + Gentamicina

Ampicilina + Gentamicina
Se dividió a las pacientes con ruptura de membranas durante la labor de acuerdo las posibilidades de infección $(17,18)$.

Los datos fueron obtenidos de las historias clínicas y registrados en un formulario, luego procesados en los programas Microsoft Excel versión 2010 y SPSS en español versión 15 .

El estudio fue revisado y aprobado por el Comité de Ética de la Universidad Peruana Cayetano Heredia.

\section{RESULTADOS}

En el periodo de estudio se realizaron 4218 cesáreas; $78(1,85 \%)$ se realizaron en periodo expulsivo. Se incluyeron 67 pacientes que cumplieron los criterios de selección; las características demográficas se

\begin{tabular}{|c|c|c|}
\hline & $\mathbf{N}^{\circ}$ & $\%$ \\
\hline \multicolumn{3}{|l|}{ Rechazo vesical } \\
\hline $\mathrm{Si}$ & 55 & 82,1 \\
\hline No & 12 & 17,9 \\
\hline \multicolumn{3}{|l|}{ Histerotomia } \\
\hline Segmentaria alta & 63 & 94,0 \\
\hline Segmentaria baja & 2 & 3,0 \\
\hline Corporal & 2 & 3,0 \\
\hline \multicolumn{3}{|l|}{ Desencajamiento } \\
\hline Abdominal & 57 & 85,1 \\
\hline Vaginal & 10 & 14,9 \\
\hline \multicolumn{3}{|c|}{ Espera de turno operatorio (min) } \\
\hline 30 minutos & 8 & 11,9 \\
\hline $31-60$ minutos & 35 & 52,2 \\
\hline $61-90$ minutos & 12 & 17,9 \\
\hline $91-120$ minutos & 12 & 17,9 \\
\hline \multicolumn{3}{|c|}{ Tiempo operatorio } \\
\hline$\leq 45$ minutos & 55 & 82,1 \\
\hline $46-90$ & 12 & 17,9 \\
\hline \multicolumn{3}{|c|}{ Dias de hospitalización post-operatorio } \\
\hline 3 - 6 días & 63 & 94,0 \\
\hline$>6$ días & 4 & 6,0 \\
\hline
\end{tabular}
muestran en la tabla 1 .

Tabla 3. Características de la técnica quirúrgica. 
Dos (3\%) pacientes tuvieron ruptura prolongada de membranas, $52(77,6 \%)$ pacientes tuvieron entre 120 y 240 minutos de periodo expulsivo, que incluyó la espera del turno operatorio. En 20 (29,9\%) pacientes la altura de presentación durante la cesárea estuvo encajada. El antibiótico profiláctico más usado fue Cefazolina en el 64,2 \% de casos, 12 (17,9\%) pacientes no recibieron antibiótico profiláctico (Tabla 2).

En $55(82,1 \%)$ pacientes se realizó rechazo vesical, en $63(94 \%)$ histerotomía segmentaria alta y en 10 $(14,9 \%)$ casos se efectuó desencajamiento de la cabeza fetal con asistencia vaginal (Tabla 3 ).

En general, 59 /67 (88,1\%) pacientes presentaron algún tipo de complicación.

Se realizaron dos transfusiones de sangre. Una en forma pre operatoria en una paciente con anemia crónica y otra en paciente complicada con atonía uterina, desgarro de segmento y laceración de vaso uterino.

En la tabla 4 se muestra las complicaciones. En el caso de desgarro de segmento con compromiso de cuerpo y laceración de arteria uterina unilateral, se

Tabla 4. Complicaciones maternas de la operación cesárea en el periodo expulsivo.

\begin{tabular}{lcr}
\hline & $\mathbf{N}^{\circ}$ & \multicolumn{1}{c}{$\mathbf{0}$} \\
\hline Inercia Uterina & & \\
Hipotónia & 8 & 11,9 \\
Atonía & 2 & 3,0 \\
Desgarro Uterino & & \\
Solo segmento & 3 & 4,5 \\
Segmento + cuerpo & 1 & 1,5 \\
Segmento + vagina & 1 & 1,5 \\
Laceración de Arteria Uterina & & \\
Unilateral & 3 & 4,5 \\
Bilateral & 1 & 1,5 \\
Hemorragia Puerperal & 4 & 6,0 \\
Trasfusión Sanguínea & 2 & 3,0 \\
Infección puerperal & & \\
Endometritis & 1 & 1,5 \\
Infección de herida operatoria & 3 & 4,5 \\
Anemia & 56 & 83,5 \\
TOTAL Complicación materna & 59 & 88,1 \\
\hline
\end{tabular}

había realizado la maniobra de desencajamiento de cabeza por vía vaginal, además se produjo hematoma de ligamento ancho unilateral.

No se produjeron lesiones en tracto urinario ni digestivo y tampoco hubo necesidad de reintervención quirúrgica.

La mayoría de pacientes, 63 (94\%) tuvieron entre 3 y 6 días de hospitalización.

\section{DISCUSIÓN}

La cesárea realizada en periodo expulsivo se considera en aumento (8), llegando hasta $3,22 \%$ de casos (19), superior a lo hallado en nuestro estudio.

Este procedimiento está enmarcado en un escenario médico legal ya que tienen entre 3,1 a 4,6 veces más riesgo de sufrir complicaciones maternas severas (6).

Las complicaciones más frecuentes relacionadas a la cesárea varían según las distintas series y circunstancias, siendo las principales la infección de herida operatoria $(0,7$ a $7,5 \%)$, la transfusión de sangre $(5,3 \%)$, la hemorragia puerperal $(3,1 \%)$, la endomiometritis $(1,6 \%$ a $8,3 \%)$, desgarro de histerorrafia, lesión urinaria e intestinal $(20,21)$.

La frecuencia de atonía uterina de la cesárea en expulsivo fue de 9\% según Sucak (11) muy superior a nuestro hallazgo (3\%). Hay que resaltar que sumados nuestros casos de atonía e hipotonía agrupan al 14,9\%.

Nuestro hallazgo de 7,5\% (5 casos) en desgarros de la histerotomía se encuentran en el rango de otras series que refieren valores porcentuales de 4,5, 15, 22 y $25 \%(12,13,14,19)$. No se describe en la literatura laceración de arterias uterinas y formación de hematomas.

Phipps (22) reporta 42 lesiones vesicales de 14 757 cesáreas (incidencia de $0,28 \%$ ), otros autores describen frecuencias de injuria de sistema urinario de la cesárea en expulsivo entre 0,087 y $7 \%(11,23)$. La frecuencia referida de la histerectomía en la cesárea en expulsivo fue de $2 \%$ (11). En nuestro estudio no se produjeron lesiones vesicales ni intestinales. Tampoco hubo necesidad de histerectomía al igual que Radha (19).

En relación a hemorragia puerperal, las mujeres sometidas a cesárea en periodo expulsivo tienen 3,1 
veces más probabilidades de sufrir la pérdida de sangre mayor de $1000 \mathrm{ml}$ y tienen 2,9 veces más probabilidades de ser trasfundidas $(3,6)$. Radha encuentra $2,7 \%$ de operadas en expulsivo con hemorragia puerperal, hallazgo muy inferior al nuestro $(5,9 \%)$, y ningún caso de necesidad de trasfusión sanguínea. Sin embargo nuestra frecuencia de transfusión (3\%), fue más baja que la encontrada por Ayhan quien describe 7\% (11).

Para Várkonyi (24), la tasa global de infecciones relacionadas a la cesárea es de $3.6 \%$, siendo mayor para las realizadas en situación de urgencia con un $11,7 \%$ (21), en nuestra muestra se pudieron encontrar frecuencias menores a las ya mencionadas (6\%), lo contrario a lo descrito por Radha (19), quien niega hallazgos de morbilidad febril en sus informes. Es importante mencionar en este punto, que en un $17.9 \%$ de nuestras pacientes no se les aplicó antibiótico profilaxis, y que casi la gran mayoría recibieron por lo menos de una a tres dosis de antibióticos.

La anemia encontrada en nuestra población fue de acuerdo a los valores de hemoglobina de control post operatoria, pero hay que suponer que es muy probable que gran parte de las pacientes ya presentaban dicha patología previa a la intervención quirúrgica, dato que es muy difícil precisar por el tipo de control prenatal que tienen las pacientes y al momento en que se toman los exámenes prenatales. Dicho sesgo idealmente se corregiría evaluando la caída real de la hemoglobina debido a la operación cesárea, es decir obteniendo la hemoglobina pre y post operatoria, lo cual no siempre se cumple.

Según Sung (14), las cesáreas duraron menos de 90 minutos en $91 \%$ de casos, en nuestra muestra se encuentra que el $100 \%$ de las cirugías duraron menos de 90 minutos, siendo el tiempo promedio calculado de 38,5 minutos.

En referencia a la espera ideal de 30 minutos del turno operatorio, $25 \%$ de los casos cumplen con esta norma según Nageotte (16), en nuestra realidad solo se cumple en el 11,9\% de los casos, siendo en el 35,8\% de pacientes la espera entre 60 y 120 minutos y 65,4 minutos en promedio.

Entre los factores limitantes podemos considerar la falta de registro de hemoglobina previa al acto quirúrgico en todos los casos, control parcial de labor de parto, uso de diferente técnica quirúrgica y manejo antibiótico, problemas que debieran corregirse mediante un estudio prospectivo.
En conclusión, la cesárea realizada en período expulsivo presenta complicaciones frecuentes, algunas de las cuales pueden ser severas.

\section{Declaración de financiamiento y de conflictos de interés:}

El estudio fue financiado por los investigadores, quienes declaran no tener conflictos de intereses.

\section{Contribución de autoría:}

JP, diseño del estudio, recolección e interpretación de los datos y elaboración del informe de investigación; JD y CC, diseño del estudio, interpretación de los datos, revisión del informe final de la investigación.

\section{Correspondencia:}

Javier Puma Medina

Correo electrónico: javier.puma.m@upch.pe

\section{REFERENCIAS BIBLIOGRÁFICAS}

1. Encarnacion B, Zlatnik MG. Cesarean delivery technique: evidence or tradition? A review of the evidence-based cesarean delivery. Obstet Gynecol Surv (Internet). 2012 (Citado el 15 de enero del 2014); 67 (8):483-94. Disponible en: http://www. ncbi.nlm.nih.gov/pubmed/22926273.

2. Pacora $\mathrm{P}$, Ingar $\mathrm{W}$, Buzzio $\mathrm{Y}$, Reyes $\mathrm{M}$, Oliveros M. Desproporción feto pélvica en un hospital de Lima: Prevalencia, consecuencias, predicción y prevención. Rev Per Ginecol Obstet. (Internet). 2007 (Citado el 15 de enero del 2014); 53:193-198. Disponible en: http://sisbib.unmsm.edu.pe/bvrevistas/ginecologia/ vol53_n3/pdf/a10v53n3.pdf

3. Alkire BC, Vincent JR, Burns CT, et al. Obstructed Labor and Caesarean Delivery: The Cost and Benefit of Surgical Intervention. 2012. PLoS ONE (Internet). 2012 (Citado el 10 de enero del 2014);7(4): e34595.); Disponible en: http://www.plosone. org/article/info\%3Adoi\%2F 10.1371\%2Fjournal. pone. 0034595

4. Landesman $\mathrm{R}$, Graber EA. Abdominovaginal delivery: modification of the cesarean section operation to facilitate delivery of the impacted head. Am J Obstet Gynecol (Internet). 1984 (Citado el 5 de enero del 2014); 148(6):707-10. Disponible en: http://www.ncbi.nlm.nih.gov/pubmed/6702937

5. Fasubaa OB, Ezechi OC, Orji EO,et al. Delivery of the impacted head of the fetus at caesarean section after prolonged obstructed labour: a randomised comparative study of two methods. J Obstet 
Gynaecol. (Internet) 2002. (Citado el 5 de enero del 2014); 22(4):375-8. Disponible en: http://www.ncbi. nlm.nih.gov/pubmed/12521457

6. Selo-Ojeme D, Sathiyathasan S, Fayyaz M. Caesarean delivery at full cervical dilatation versus caesarean delivery in the first stage of labour: comparison of maternal and perinatal morbidity. Archives of Gynecology and Obstetrics (Internet). 2008 (Citado el 10 de enero del 2014); 278 (3): 245-249. Disponible en: http://link.springer.com/ article/10.1007/s00404-007-0548-5

7. Rabiu KA, Adewunmi AA, Akinola OI, Eti AE, Tayo AO. Comparison of maternal and neonatal outcomes following caesarean section in second versus first stage of labour in a Tertiary Hospital in Nigeria. Niger Postgrad Med J (Internet). 2011 (Citado el 10 de enero del 2014); 18: 165-171. Disponible en: https://www.thieme-connect.de/ejournals/pdf/ 10.1055/s-0032-1333411.pdf

8. Sethuram R, Jamjute P, Kevelighan E, Delivery of the deeply engaged head: a lacuna in training. J Obstet Gynaecol. (Internet). 2010 (Citado el 20 de diciembre del 2013); 30(6):545-9. Disponible en: http://www. ncbi.nlm.nih.gov/pubmed/20701498

9. Unterscheider J, McMenamin M, Cullinane F. Rising rates of caesarean deliveries at full cervical dilatation: a concerning trend. Eur J Obstet Gynecol Reprod Biol (Internet). 2011 (Citado el 20 de diciembre del 2013); 157(2):141-4. Epub 2011 Apr 5. Disponible en: http:// www.ncbi.nlm.nih.gov/pubmed/21470764.

10. Corona AA, Higueras T, Cabero L1. Complicaciones maternas a corto plazo en pacientes con cesárea. Progresos de obstetricia y ginecología: revista oficial de la Sociedad española de ginecología y obstetricia (Internet). 2008 (Citado el 16 de diciembre del 2013); 51(12): 703-708. Disponible en: http://dialnet. unirioja.es/servlet/articulo? codigo $=2782386$.

11. Sucak A, Celen S, Akbaba E, Soysal S, Moraloglu O, Danışman N. Comparison of nulliparas undergoing cesarean section in first and second stages of labour: a prospective study in a tertiary teaching hospital. Obstet Gynecol Int (Internet). 2011(Citado el 16 de diciembre del 2013); 2011. Epub 2011 Sep 20. Disponible en: http://www.ncbi.nlm.nih.gov pubmed/ 21941557

12. Chopra S, Bagga R, Keepanasseril A, Jain V, Kalra J, Suri V. Disengagement of the deeply engaged fetal head during cesarean section in advanced labor: conventional method versus reverse breech extraction. Acta Obstet Gynecol Scand (Internet). 2009 (Citado el 13 de diciembre del 2013); 88(10): 11636. Disponible en: http://www.ncbi.nlm.nih.gov/ pubmed/19925377

13. De la Torre L, González-Quintero VH, et al. Significance of accidental extensions in the lower uterine segment during cesarean delivery. Am J Obstet
Gynecol (Internet). 2006 (Citado el 16 de diciembre del 2013); 194(5):e4-6. Epub 2006 Apr 21. Disponible en: http:/www.ncbi.nlm.nih.gov/pubmed/ 16647896

14. Sung JF, Daniels KI, Brodzinsky L, El-Sayed YY, Caughey AB, Lyell DJ. Cesarean delivery outcomes after a prolonged second stage of labor. Am J Obstet Gynecol (Internet). 2007 (Citado el 16 de diciembre del 2013); 197(3):306.e1-5. Disponible en: http:// www.ncbi.nlm.nih.gov/pubmed/17826431

15. Hilton P. Vésico-vaginal fistulas in developing countries. Int J Gynaecol Obstet (Internet). 2003 (Citado el 16 de diciembre del 2013); 82(3):28595. Disponible en: http:/www.ncbi.nlm.nih.gov/ pubmed/14499975

16. Nageotte MP, Vander Wal B. Achievement of the 30-minute standard in obstetrics-can it be done? Am J Obstet Gynecol (Internet). 2012 (Citado el 16 de diciembre del 2013); 206(2):104-7. Disponible en: http:/www.ncbi.nlm.nih.gov/pubmed/22000896

17. Vigil-De Gracia P, Savransky R, Pérez Wuff JA, Delgado Gutiérrez J, Nunez de Morais E. Ruptura Prematura de Membranas. FLASOG, 2011:1. (Citado el 13 de enero de 2014). Disponible en: http:/www. sogiba.org.ar/novedades/GC1RPM.pdf

18. Mercer B. Premature Rupture of the Membranes. En: Protocols for High-Risk Pregnancies. Oxford, WileyBlackwell, 2010:448-460.

19. Radha P, Tagore S, Rahman MF, Tee J. Maternal and perinatal morbidity after Caesarean delivery at full cervical dilatation. Singapore Med J (Internet). 2012. (Citado el 16 de diciembre del 2013); 53(10):655-8. Disponible en: http://www.ncbi.nlm. nih.gov/pubmed/23112016

20. Corona A, Higueras T, Cabero Ll. Complicaciones maternas a corto plazo en pacientes con cesárea. Progresos de obstetricia y ginecología. (Internet). 2008;51(12) (Citado el 13 de enero del 2014); 703708. Disponible en: http://dialnet.unirioja.es/servlet/ articulo? codigo $=2782386$

21. Monroy M, Torreblanca M. Comparación de la frecuencia de endometritis e infección de herida operatoria en cesáreas programadas y de urgencia. Rev Per Ginecol Obstet (Internet). 2004 (Citado el 16 de diciembre del 2013); 50:166-170. Disponible en: http://sisbib.unmsm.edu.pe/bvrevistas/ginecologia/ vol50_n3/pdf/A04V50N3.pdf

22. Phipps MG, Watabe B, Clemons JL, Weitzen S, Myers DL. Risk factors for bladder injury during cesarean delivery. Obstet Gynecol. (Internet). 2005 (Citado el 16 de diciembre del 2013); 105(1):156-60. Disponible en: http:/www.ncbi.nlm.nih.gov/pubmed/15625157

23. Alcocer UJ, Bonilla MM, Gorbea ChV, Velázquez VB. Risk factors for bladder injuries during cesarean section. Actas Urol Esp (Internet). 2009 (Citado el 16 de diciembre del 2013); 33(7):806-10. Disponible en: http://www.ncbi.nlm.nih.gov/pubmed/19757667 . 
24. Várkonyi I, Makai I, Papdiné Nyíri G, Bacskó G, Kardos L. Postoperative surveillance of wound infection after cesarean section at Kenézy Hospital, Debrecen, Hungary. Orv Hetil. (Internet). 2011 (Citado el 16 de diciembre del 2013); 152(1):14-22. Disponible en: http:/www.ncbi.nlm.nih.gov/pubmed/21177226 .

Recibido: 12/02/2014

Aceptado: 28/10/2014 